\title{
Development of knowledge bases and the reliability of a decision support system for behavioral treatment consultation for persons with mental retardation: The Mental Retardation-Expert
}

\author{
MATTHEW G. HILE \\ Missouri Institute of Mental Health, St. Louis, Missouri \\ DONNA M. CAMPBELL \\ Webster University, St. Louis, Missouri \\ BAGHER B. GHOBARY \\ Missouri Institute of Mental Health, St. Louis, Missouri \\ and \\ MARCIE N. DESROCHERS \\ College of Charleston, Charleston, South Carolina
}

\begin{abstract}
The development of expert and decision support systems requires the collection, organization, codification, and storage of a body of specialized knowledge. The development, using reconstructive methods, of two such knowledge bases, the first containing the current scientific literature and the second containing an expert's knowledge, is described for an automated decision support system, the Mental Retardation-Expert. This system provides practitioners with assistance in the treatment of aggressive, self-injurious, and destructive behaviors displayed by individuals with mental retardation or developmental disabilities. The average interobserver reliability of the expert knowledge base ranged from $92.5 \%$ to $95.0 \%$ when calculated across four clinicians' assessments of 31 abstracted cases.
\end{abstract}

The provision of effective treatment in residential centers that serve individuals who are mentally retarded or developmentally disabled is becoming more and more difficult. Deinstitutionalization has moved many individuals from these centers into less restrictive community settings. Those who remain often have significant behavioral problems that are unacceptable in their communities (Borthwick-Duffy, Eyman, \& White, 1987). Such problems include aggressive, self-injurious, and destructive forms of behavior. Of the individuals left in public or community residential facilities, some $53 \%-84 \%$ exhibit moderate-to-severe behavioral problems (Calkins et al., 1983; Eyman \& Borthwick, 1980; Fovel, Lash, Barron, \& Roberts, 1989).

In part because of the difficult client population, these centers have high staff turnover rates (Grossman, 1984), which result in young and relatively inexperienced staffs.

This work is supported in part by Grant 1 R29 MH43439 from the National Institute of Mental Health. The opinions expressed in this paper are those of the authors, and no official endorsement by any agency should be inferred. The authors gratefully acknowledge the assistance of Stephanie Patag and Bruce Vieweg. Correspondence should be addressed to M. G. Hile, Missouri Institute of Mental Health, 5247 Fyler Avenue, St. Louis, MO 63139-1494 (e-mail: medmip@mizzou1).
In addition, because of budget reductions, fewer people are being asked to provide increasing levels of service to the individuals with higher levels of needs (Scheerenberger, 1982). This leaves staffs with little time to assemble the information necessary to create effective interventions and little time to keep up with the large volume of current scientific literature (Bostwick, 1985) that contains the latest techniques and approaches to treatment.

Readily accessible consultation, treatment planning assistance, and scientific information would potentially support these staffs in their daily tasks. An automated decision support system (DSS) is one way to provide such assistance. A DSS is a computer program designed to support effective and efficient problem solving (Te'eni \& Ginzberg, 1991). Such a system can include a wide variety of tasks necessary to support its users. In residential settings, for persons with mental retardation, such a system might suggest various assessment options to be explored, advise users about available treatment procedures, provide reminders of important information to collect or concepts to keep in mind, teach individuals how to solve specific problems, support or confirm a user's treatment or analysis decision, and so on. The components of such a system would be limited only by the needs of its users. 
The Mental Retardation-Expert (MR-E, pronounced "Murry") is a DSS designed to assist clinicians who work with mentally retarded or developmentally disabled individuals who exhibit clinically significant inappropriate behaviors. MR-E supports clinicians by providing several services including expert-based behavioral consultation, on-line literature reviews and annotations, a comprehensive behavioral glossary, and a set of exemplary behavioral treatment programs. Because of the central role that knowledge bases play in DSSs, in this paper we will describe the development and structure of two of MR-E's knowledge bases, one that contains the current scientific literature, and one that contains information from experts' knowledge of treatments. Each of these knowledge bases represents a different approach to knowledge-base construction. The first comprises freely available published literature; the second, interviews, transcripts, and protocols.

A knowledge base is a collection of facts tied together with specific rules about how these facts relate to one another. The structure and content of this knowledge base determine the abilities and limitations of the DSS. Essentially, the knowledge base is a model of the structure, content, and decision-making processes inherent in a particular area (Carrol \& Olson, 1988). Authentic and reconstructive techniques are two prominent ways to construct a knowledge base. Authentic techniques model the expert's actions and decisions without regard to the underlying reasoning. This is a "black box" approach, concerned only with duplicating the expert's decisions. Alternatively, reconstructive methods seek to understand the specific reason why a particular decision is made and to model that underlying process. This approach to knowledge-base construction draws upon consensus and public understanding to arrive at its decisions. Although both approaches were used in its development, MR-E relies heavily on reconstructive methods.

\section{Literature Knowledge Base}

The literature knowledge base was developed with a reconstructive approach. It is reconstructive in that in publications authors examine their behavior and construct explanations of those actions. Knowledge from the literature, embedded in MR-E, allows users to access current scientific thinking independently of the knowledge included in the rest of the system. This knowledge base contains specific reviews of the literature, annotations about individual papers, and a comprehensive behavioral glossary.

The development of the literature knowledge base required a pool of relevant literature. This consisted of a database containing all the available articles on the treatment of clinically significant problematic behaviors in persons with mental retardation or developmental disabilities. Initially, various electronic searches were conducted and the identified literature was obtained. Additional references were collected by examining the bibliographies of the reviews of the literature in the area as well as the bibliographies of any article published within the last 10 years. A research assistant entered these articles with ap- propriate key terms and a brief annotation. This database, which currently contains more than 3,600 citations, serves as the basis of the literature knowledge base.

The collected data are organized into the knowledge base manually. Essentially, treatment-oriented questions are posed, and the literature is searched for answers. These answers are then summarized and included in the knowledge base. For example, the first treatment-oriented question addressed self-injurious biting in individuals with mental retardation. The database was culled to collect all of the articles written over a 20 -year period. The articles were abstracted, summarized, and reported as describing a wide variety of treatment possibilities. Then a traditional comprehensive review of this literature was written (Hile \& Vatterott, 1991). The review was in turn used as the basis for an electronic version that is both simpler and more complete than the printed version. It is simpler because a variety of details included in the printed version were removed from the body of the paper and placed in hypertext references, which are essentially electronic footnotes. If the user wishes additional information-as, for example, on the way in which the initial literature search was conducted-the click of a mouse button will display a few paragraphs describing that process. With this method, the user can read the essential information and obtain only the desired details. The electronic version is more complete because it also contains hypertext links to the complete annotations of each cited article. Thus, the user may easily access additional information about a particular study.

Abstracting information from the published literature to create knowledge bases has several important advantages over other methods of knowledge-base development. Information in the literature is easily obtainable and is formatted in a way that makes it easy to understand. Typically, published literature has undergone some degree of peer review and should, as a result, be scientifically sound. It is verifiable and may have been corroborated in other publications. It may be research oriented, providing information on competing hypotheses and interpretations. In addition, it covers a wide range of problems, clients, and treatment options from widely disparate researchers and clients.

Nonetheless, there are some disadvantages to using the published literature in this reconstructed form. First, the amount of information contained in any single article is limited. Because of the limited space available in many journals, certain important contextual information concerning clients and treatment may be missing. Publications are written to make a particular point (e.g., the clarification of an individual treatment approach), not necessarily to detail the various important contextual variables. Consistency among articles is also a major difficulty. It is difficult or impossible to find two articles that agree exactly on any particular point. Though peer review does serve to increase the quality of published materials, the process does not necessarily eliminate inadequate or confusing information. In addition, using the published literature to develop knowledge bases is a very time- 
consuming process. The literature must be collected, read, understood, abstracted, and summarized in a way that is helpful to users and, at the same time, relevant to the knowledge base.

Although there are both advantages and disadvantages to this method of developing a knowledge base, it does provide users with information that would otherwise, because of its form and overall lack of summative structure, be much less accessible. By reconstructing this information from the literature, we can share concepts and facts, thereby allowing users to make judgments independent of any individual expert. When used together with MR-E's other components, users have access to a rich source of assistance in understanding their individual clients and in developing effective treatment protocols. Indeed, MR-E's users find the literature knowledge base to be particularly helpful, and they frequently suggest additions and extensions to fit their individual needs.

\section{Expert Knowledge Base}

The human expertise embodied in MR-E provides clinicians with assistance in developing behavioral treatment programs. To do this, it leads the practitioner through a functional assessment of a single client designed to help the clinician identify the function or purpose of the specific behavior problem. For example, aggression may function to delay the individual's participation in a disliked activity or alternatively to gain attention (albeit negative) from a particular person. On the basis of the functional assessment, the clinician is offered a variety of treatment suggestions, emphasizing the development of adaptive skills, which may be incorporated into an individualized treatment protocol.

MR-E's functional consultation is modeled on the knowledge of an expert in the treatment of clinically significant inappropriate behaviors. Data for the knowledge base were collected by using two approaches. One approach involved the observation of behavioral consultations between the expert and clinicians. The second combined the expert's written treatment logic protocol with taped interviews regarding treatment selection logic and suggestions.

Initially, observations of actual consultations were planned as the basis of the expert knowledge base, an authentic method. The advantages of such an analysis include the ability to see the behaviors that are to be modeled in the system, to capture the richness of interaction between expert and novice, and to discern what the expert does without need of reconstructive interpretation. Yet, in the development of MR-E, the disadvantages of this approach outweighed these advantages. A major stumbling block involved the confidentiality of the clients being discussed. Because of this concern, the participants were reticent to allow the recording of consultations that was necessary for later analysis. However, a more important practical difficulty was the relatively limited range of naturally occurring problems available for consultation. Given the breadth of possible functional interpreta- tions, it became obvious that there would be an insufficient range of available cases on which to base a system. Since the knowledge base had to represent a broad spectrum of problems, it became necessary to use a different approach to gather the necessary knowledge.

The second approach, the use of reconstructive techniques, rested on a written treatment decision protocol developed by the expert. That protocol was used to construct a set of IF-THEN rules that served as the basis of the knowledge base. Within the context of this initial set of rules, the expert and the developer would meet and discuss different clients, behavioral problems, and specific rules. Information from the resulting transcripts was used to make changes in the knowledge base to more closely fit the expert's treatment consultation approach and behavioral treatment theory. Feedback from individuals testing MR-E was also used to obtain additional information and clarification from the expert and to change the knowledge base to reflect the users' needs. Information from the literature, including examples, suggestions, and treatments, was added. The resulting knowledge base contains more than 230 structured rules within 21 functional areas. It contains general information about how treatment problems are to be solved, as well as information about specific treatment strategies.

This reconstructive method has the disadvantage of requiring experts to describe what they do, an activity that is often very difficult (Johnson, 1983). Its advantages for MR-E include the ability to address a variety of problems and functions, a framework on which to base discussions with the expert, a theoretically linked set of information, relatively straightforward development, a format consistent with consultation (discussions with a novice), and a structure that can be empirically tested.

\section{Reliability}

The reliability of the consultation knowledge base was evaluated by comparing the consultation results obtained by four clinician users across 31 cases that had been abstracted from the literature. These clinicians were instructed to "read the case and try to place yourself in the role of the clinician while answering the questions. Try to stay as close to the available data as possible though you may make some educated guesses and hypotheses." To assess rater similarity, an interobserver agreement (IOR) score was computed to compare each rater with the other three by dividing the number of agreements by the number of agreements plus disagreements and multiplying by 100 (Kazdin, 1982, p. 54). These scores were computed on the observers' ratings of the 21 functional areas. The level of agreement between raters was uniformly high. The average IOR ranged from a low of $92.5 \%$ to a high of $95.0 \%$. Furthermore, when all major hypotheses were considered ( 31 cases with 21 hypotheses per case, $N=651$ ), all four raters agreed $78 \%$ of the time and three or more raters agreed $95 \%$ of the time. These results show that, when presented with the same information, independent raters can reliably use MR-E 
to obtain similar functional analyses and, by extension, similar treatment recommendations.

\section{Summary}

Authentic and reconstructive methods of knowledgebase development each have advantages and disadvantages. In the development of two knowledge bases for MR-E, we relied primarily on reconstructive techniques. The literature knowledge base has succeeded in providing users with information that has been difficult or impossible for them to obtain, and their requests for additions and extensions suggest that it fills a perceived need. The reconstructive method has also been used successfully to develop a reliable system for evaluating the function of specific undesirable behaviors. However, these are clearly only the initial stages of evaluation. The utility and validity of both knowledge bases will have to be carefully assessed over the course of their use.

\section{REFERENCES}

Borthwick-Duffy, S. A., Eyman, R., \& White, J. (1987). Client characteristics and residential placement patterns. American Journal of Mental Deficiency, 92, 24-30.

Bostwick, G. J. (1985). Knowledge utilization and decision support systems in family therapy. In C. R. Figley (Ed.), Computers and family therapy (pp. 153-165). New York: Haworth.

Calkins, C. F., Krantz, S., McGreevy, P., Neff, F. W., Griggs, P., \& INTAGLIATA, J. (1983). The residential placement study: A descriptive study of client needs and residential services for the developmentally disabled in Missouri. Kansas City, MO: University of Missouri, University Affiliated Facility for Developmental Disabilities.

Carrol, J. M., \& Olson, J. R. (1988). Mental models in humancomputer interaction. In M. Helander (Ed.), Handbook of humancomputer interaction (pp. 45-66). New York: North-Holland.

Eyman, R. K., \& BorTHWick, S. A. (1980). Patterns of care for mentally retarded persons. Mental Retardation, 18, 63-66.

Fovel, J. T., Lash, P. S., Barron, D. A., \& Roberts, M. S. (1989). A survey of self-restraint, self-injury, and other maladaptive behaviors in an institutionalized retarded population. Research in Developmental Disabilities, 10, 377-382.

Grossman, H. (1984). Professional responsibilities. Mental Retardation, 22, 212-217.

Hile, M. G., \& VATterott, M. (1991). Two decades of treatment for self-injurious biting in individuals with mental retardation or developmental disabilities: A treatment focused review of the literature. Joumal of Developmental \& Physical Disabilities, 3, 81-113.

JoHnson, P. (1983). What kind of expert should a system be? Journal of Medicine \& Philosophy, 8, 77-97.

KAZDIN, A. (1982). Single-case research design: Methods for clinical and applied settings. New York: Oxford University Press.

SCheERenberger, R. (1982). Public residential services, 1981: Status and trends. Mental Retardation, 20, 210-215.

TE'ENI, D., \& GinzberG, M. J. (1991). Human-computer decision systems: The multiple roles of DSS. European Journal of Operational Research, 50, 127-139. 